\title{
A Stochastic Dual Dynamic Programming Approach for Optimal Operation of DER Aggregators
}

\author{
Panagiotis Fatouros, Student Member, IEEE, Ioannis Konstantelos, Member, IEEE, \\ Dimitrios Papadaskalopoulos, Member, IEEE, and Goran Strbac, Member, IEEE \\ Department of Electrical and Electronic Engineering, \\ Imperial College London \\ London, UK
}

\begin{abstract}
The operation of aggregators of distributed energy resources (DER) is a highly complex task that is affected by numerous factors of uncertainty such as renewables injections, load levels and market conditions. However, traditional stochastic programming approaches neglect information around temporal dependency of the uncertain variables due to computational tractability limitations. This paper proposes a novel stochastic dual dynamic programming (SDDP) approach for the optimal operation of a DER aggregator. The traditional SDDP framework is extended to capture temporal dependency of the uncertain wind power output, through the integration of an n-order autoregressive (AR) model. This method is demonstrated to achieve a better trade-off between solution efficiency and computational time requirements compared to traditional stochastic programming approaches based on the use of scenario trees.
\end{abstract}

Index Terms-Aggregators, autoregressive modelling, distributed energy resources, stochastic dual dynamic programming, uncertainty.

\section{NOMENCLATURE}

A. Indexes

$t \quad$ Index of time periods, running from 1 to $T$.

$b \quad$ Index of energy storage (ES) units, running from 1 to $B$.

$f \quad$ Index of flexible loads (FL), running from 1 to $F$.

$w \quad$ Index of wind turbines (WT), running from 1 to $W$.

$m \quad$ Index of micro-generators, running from 1 to $M$.

B. Parameters

$C_{t}^{\text {grid }} \quad$ Grid price at period $t$.

$C_{m}^{g e n} \quad$ Operating cost of micro-generator $m$.

$C^{\text {dem }}$ Cost of demand shedding.

$E_{0, b} \quad$ Initial energy level of ES unit $b$.

$\eta_{b} \quad$ Round-trip efficiency of ES unit $b$.

$E_{b}^{\max } \quad$ Maximum energy level of ES unit $b$.

$E_{b}^{\text {min }} \quad$ Minimum energy level of ES unit $b$.

$P_{b}^{S} \quad$ Maximum power rating of ES unit $b$.

$D_{t, f}^{\text {base }} \quad$ Baseline demand of FL $f$ at period $t$.
$S_{f} \quad$ Load shifting limit of FL $f$ at period $t$.

$P_{t}^{g, \text { sell }}$ Maximum power sold to the grid at period $t$.

$P_{t}^{g, b u y}$ Maximum power bought from the grid at period $t$.

$P_{m}^{g e n} \quad$ Maximum power rating of micro-generator $m$.

$P_{t, w}^{w i n d} \quad$ Available wind power output of WT $w$ at period $t$.

$P_{t-n, w}^{\text {wind }} \quad$ Available wind power output of WT $w, n$ periods before $t$.

$\varphi_{n, w} \quad n^{\text {th }}$ autoregressive coefficient associated with power output of WT $w$.

$\varepsilon_{t, w} \quad$ White noise term associated with power output of WT $w$ at period $t$.

$D_{t}^{\text {inf }} \quad$ Inflexible demand at period $t$.

$\Delta t \quad$ Period $t$ duration.

C. Variables

$p_{t}^{g r i d} \quad$ Power sold to (positive)/bought from (negative) the grid at period $t$.

$p_{t, m}^{g e n} \quad$ Power output of micro-generator $m$ at period $t$.

$p_{t}^{d e m} \quad$ Demand shed at period $t$.

$e_{t, b} \quad$ Energy level of ES unit $b$ at period $t$.

$p_{t, b}^{s} \quad$ Power input (positive) / output (negative) of ES unit $b$ at period $t$.

$d_{t, f}^{s h} \quad$ Change of demand of FL $f$ due to load shifting at period $t$.

$d_{t, f}^{\text {total }}$ Demand of FL $f$ after load shifting at period $t$.

$p_{t, w}^{\text {wind }}$ Dispatched wind power output of turbine $w$ at period $t$.

\section{INTRODUCTION}

\section{A. Background}

The envisaged large-scale introduction of renewable energy sources (RES) in future power systems, along with the intensified efforts towards the electrification of the heating and transport sectors, have enhanced the value of distributed energy resources (DER), such as flexible loads, controllable 
micro-generators and energy storage [1]. The capability of these resources to modify their consumption or production profiles can facilitate the economic and environmental performance of the system. However, the small individual size and inherent unpredictability characterizing such resources combined with their continuously increasing penetration in power systems have complicated the task of optimal scheduling and coordination. This paradigm change has triggered the introduction of DER aggregators, which pool a significant number of DERs, aggregate their flexibility and coordinate their operation [1].

However, the operation of a DER aggregator involves significant complexity, as it involves the optimal coordination of a large number of diverse DER with time-coupled characteristics. Furthermore, it is subject to numerous and diverse factors of uncertainty, associated with both the operation of its DER portfolio (e.g. output of renewable micro-generators, consumers' preferences and requirements) as well as market conditions (e.g. wholesale energy prices). As a result, identifying the optimal aggregator market strategies constitutes a multi-stage stochastic problem, characterized by a large number of decision variables and constraints. Approaches in the existing literature exhibit serious limitations in dealing effectively with such high dimensional problems within reasonable computational times.

In the past, research efforts have focused primarily on classic stochastic programming approaches and the use of scenario generation and reduction techniques to capture resource variability. In [2], a random error term is added to the expected values of the energy price and conventional demand to represent their uncertain nature in the portfolio optimization of an aggregator participating in the forward and spot markets. In [3], the profit maximizing policy of a microgrid aggregator is derived, where scenarios about uncertain system load, wind power output and electricity prices are derived by MonteCarlo sampling. Finally, in [4] the problem of optimizing the energy procurement schedule of a DER aggregator under uncertain energy prices, load and RES output, is addressed. A small number of scenarios is obtained, assuming a normal probability distribution function, and a scenario reduction technique is applied to reduce the combined scenario set. In [5], a customized SDDP algorithm is presented and implemented in a microgrid context, where uncertain wind output, demand and energy price are captured employing a scenario tree model. Nodal values of the scenario tree are derived from a multivariate truncated normal distribution and temporal independence is assumed. In all previous works, temporal dependency of the uncertain variables is not explicitly considered and is poorly captured; in other words, the impact of current realizations of the uncertain variables on their future evolution is underestimated. This means that the requirement of computational tractability inhibits the incorporation of sophisticated uncertainty models; potentially significant time-dependencies are not considered, focusing instead on a limited set of scenarios for the next hour.

On the other hand, robust optimization (RO) techniques, instead of relying on a set of possible scenarios, introduce uncertainty intervals and aim to identify solutions which both ensure feasibility and are near-optimal for all scenarios within the defined bounds [6]. In [7], a robust optimization approach is proposed for the optimal energy management of a cluster of flexible loads under uncertainty in RES output and energy price. Historical data and a forecasting tool based on autoregressive models are employed to identify upper and lower bounds within a given confidence interval for the uncertain variables. Finally, in [8], the problem of managing demand response for a group of residential appliances, when uncertain electricity prices are considered, is evaluated through robust optimization. The required uncertainty intervals are derived either from a price forecasting model or as a percentage around the expected value, if such a model is not available. Even though RO can capture to some extent time dependencies between uncertain variables and expand the scenario set into consideration, it can lead to very conservative policies by enforcing feasibility constraints for each scenario and adapt the optimal decisions to the worst-case scenarios [8]-[9].

\section{B. Contributions}

In order to overcome the 'curse of dimensionality' characterizing high-dimensional problems, which arises from the need to consider temporal dependence of stochastic time series, this paper proposes a novel approach based on stochastic dual dynamic programming (SDDP). The latter was introduced in [10] for the optimal scheduling of a hydrothermal generation system, driven by the need to model the reservoir interconnections for the future inflow sequences. The ability of SDDP to refine the solution quality around areas of the state space most likely to occur ('areas of interest') facilitates the solution of high dimensional problems. The algorithm employs the dual variables of the problem to build a piecewise linear representation of the future cost function during each period without the need to discretize the state space, thus significantly reducing the problem complexity without compromising on the uncertainty representation quality. In [11], the possibility of representing stochastic processes with probability distributions conditioned on previous realizations of the same variable has been theoretically explored.

The contribution of this paper lies in developing an SDDP approach for the optimal operation of a DER aggregator, which can capture temporal dependencies for the uncertain wind power output through the integration of an autoregressive model of order $n$. Thus, the uncertainty model becomes an internal part of the stochastic problem and the solution process is explicitly driven by the impact of the stochastic variable on the problem itself instead of relying on scenario reduction techniques, which can potentially ignore important scenarios. Even though we focus on the effect of wind output uncertainty on the aggregator operation, the application of the proposed model for other types of uncertain factors is straightforward. This approach is demonstrated to achieve a better trade-off between solution efficiency and computational time requirements compared to traditional stochastic programming approaches. The comparison of the solution efficiency of the two approaches is carried out through a Monte-Carlo out-of-sample validation of the obtained $1^{\text {st }}$ stage solutions, where realizations are drawn from the true distribution of the stochastic process. 
The rest of this paper is organized as follows: Section II describes the DER aggregator model. The basic principles of the SDDP algorithm and the novel extension for modeling temporal dependencies of uncertain variables are shown in Section III. The application of the developed model on the examined DER aggregator problem and results are presented in Section IV. Finally, Section V discusses conclusions and future extensions of this work.

\section{PROBLEM DEFINITION}

We focus on the problem of the optimal operation of a DER aggregator. This entity coordinates a cluster of flexible and inflexible resources and has to come up with an optimal set of decisions at each time period of the planning horizon, while facing uncertainty regarding the available wind power output. The main complexity lies in the time dependency of the uncertain variable, since the wind output at each timeperiod depends on the observed wind output at the previous time periods. As the planning horizon expands, even a modest number of wind output realizations per period significantly increase the combinations across the whole horizon and the size of the problem grows exponentially. Fundamentally, this is a multi-stage stochastic problem with recourse, where the stochastic outputs are gradually revealed and decisions are made considering both the already observed outputs and the expected ones. Consequently, an optimal policy, which will guarantee the least-cost resource scheduling taking into account the time-dependent uncertainty at reasonable computational time, has to be derived.

In this paper we assume that the aggregator manages a number of wind turbines with uncertain output, a group of flexible and inflexible loads, a group of energy storage units and a micro-generator. Additionally, energy can be bought/sold from/to the grid and wind output can be curtailed if required. Therefore, the aggregator must identify the use of the available wind output, the amount of energy that will be bought/sold from/to the grid, the flexible loads' consumption, the storage units' charging/discharging schedule and the utilization of the micro-generator at each time period, in order to minimize the overall cost. For a particular operation horizon $t=\{1, \ldots, T\}$, the objective function comprises of the cost of the energy transactions (procuring or providing) with the grid and the cost of using the micro-generator to cover the energy needs of the flexible and inflexible loads:

$$
\begin{aligned}
& C_{\text {total }}=\min _{p_{\text {grid }} p^{\text {gen }}, p^{\text {dem }}}\left\{\sum _ { t , m } \left[\left(-p_{t}^{\text {grid }} \cdot C_{t}^{\text {grid }}+\right.\right.\right. \\
& \left.\left.\left.p_{t, m}^{\text {gen }} \cdot C_{m}^{\text {gen }}+p_{t}^{\text {dem }} \cdot C^{\text {dem }}\right) \cdot \Delta t\right]\right\}
\end{aligned}
$$

The cost of importing and exporting energy from/to the grid has been assumed to be the same for simplicity purposes. The optimal aggregator cost can be either positive or negative, depending on the size of demand, the available RES output and the flexibility of its portfolio. The aggregator should exploit the flexibility provided by the energy storage units and the flexible loads, taking into consideration the effect of wind uncertainty. The energy balance of the energy storage units is modelled in (2).

$$
e_{t, b}=\left\{\begin{array}{c}
E_{0, b} \cdot \eta_{b}+p_{t, b}^{s} \cdot \Delta t, \quad \text { if } t=1 \\
e_{t-1, b} \cdot \eta_{b}+p_{t, b}^{s} \cdot \Delta t, \quad \text { if } 1<t \leq T
\end{array} \quad \forall b\right.
$$

Constraints (3) - (4) impose limits for both electrical power and energy levels of the battery, while energy neutrality for the entire planning horizon is preserved through (5).

$$
\begin{array}{cc}
E_{b}^{\min } \leq e_{t, b} \leq E_{b}^{\max } & \forall t, b \\
-P_{b}^{S} \leq p_{t, b}^{S} \leq P_{b}^{S} & \forall t, b \\
e_{T, b}=E_{0, b} & \forall b
\end{array}
$$

Additionally, a group of different flexible loads, characterized by diverse operational features, is part of the aggregator's portfolio. A generic model for the flexible load, which can defer consumption across time if energy needs are covered during the planning horizon, has been adopted and modelled with (6) - (8):

$$
\begin{array}{cc}
d_{t, f}^{\text {total }}=D_{t, f}^{\text {base }}+d_{t, f}^{s h} & \forall t, f \\
-S_{f} \cdot D_{t, f}^{\text {base }} \leq d_{t, f}^{s h} \leq S_{f} \cdot D_{t, f}^{\text {base }} & \forall t, f \\
\sum_{t} d_{t, f}^{s h}=0 & \forall f
\end{array}
$$

Moreover, (9) - (11) impose limits on the power available from the wind turbines, the grid and the micro-generators respectively. Finally, power balance is expressed in (12).

$$
\begin{array}{cc}
p_{t, w}^{\text {wind }} \leq P_{t, w}^{\text {wind }} & \forall t, w \\
-P_{t}^{g, b u y} \leq p_{t}^{\text {grid }} \leq P_{t}^{\text {g,sell }} & \forall t \\
p_{t, m}^{\text {gen }} \leq P_{m}^{\text {gen }} & \forall t, m \\
\sum_{b} p_{t, b}^{s}-\sum_{w} p_{t, w}^{\text {wind }}+p_{t}^{\text {grid }} & \\
+\sum_{f} d_{t, f}^{\text {total }}+D_{t}^{\text {inf }}-p_{t, m}^{\text {gen }}=0 & \forall t
\end{array}
$$

\section{STOChastic Dual Dynamic Programming}

\section{A. General Formulation}

The key principle of SDDP is that the original multi-stage stochastic problem can be decomposed into a series of master problems and sub-problems, with the use of appropriate dual variables. The master problems minimize the costs incurred by the immediate decisions at each period, while the respective sub-problems minimize the future cost at future periods, given the optimal decisions of the respective master problem. This is expressed by (13) - (14), where $e_{t-1, b}$ and $d_{t-1, f}^{a g g}$ are the state variables of the stochastic problem, $C_{t}$ is the master problem cost at period $t$ and $\alpha_{t}$ corresponds to the future cost function for periods $t+1$ up to $T$.

$$
\begin{gathered}
F C_{t-1}\left(e_{t-1, b}, d_{t-1, f}^{a g g}\right)=\min \left[C_{t}+\alpha_{t}\left(e_{t, b}, d_{t, f}^{a g g}\right)\right] \\
C_{t}=\left(-p_{t}^{g r i d} \cdot C_{t}^{g r i d}+p_{t, m}^{g e n} \cdot C_{m}^{g e n}+p_{t}^{d e m} \cdot C^{d e m}\right) \cdot \Delta t
\end{gathered}
$$

In (13), the objective function of master problem for period $t$ is the minimization of the sum of current period's cost $C_{t}$ and an approximation of the cost of the remaining hours $\alpha_{t}$ obtained by solving the sub-problem which corresponds to $\alpha_{t}$. Then, for the next period, $\alpha_{t}$ is the current master problem while $\alpha_{t+1}$ is the respective sub-problem. We observe that a single-stage problem acts both as a master problem for period $t$ and as a sub-problem for $t-1$.

According to the SDDP algorithm, an iterative process which involves the successive solution of all the master 
problems (forward pass) and then all the sub-problems (backward pass), is initiated. During each iteration, a set of linear constraints ('cuts') is gradually built to provide a finer piecewise linear approximation of the future cost function (sub-problem) for each period. Each linear constraint is expressed in terms of the change in the sub-problem's optimal objective function value with respect to the master problem's state variables, as shown in (15).

$$
\begin{aligned}
\alpha_{t}^{i}\left(e_{t, b}, d_{t, f}^{a g g}\right) \geq \alpha_{t, k}^{i-1}\left(e_{t, b, i-1, k}, d_{t, f, i-1, k}^{a g g}\right) & \\
& +\sum_{h} \lambda_{t, b, i-1, k}^{E} \cdot\left(e_{t, b}-e_{t, b, i-1, k}\right) \\
& +\sum_{f} \lambda_{t, f, i-1, k}^{D} \cdot\left(d_{t, f}^{a g g}-d_{t, f, i-1, k}^{a g g}\right)
\end{aligned} \quad \forall k
$$

\section{B. Forward Pass}

The first step of the iterative simulation process is the forward pass calculation, where the master problems for periods 1 to $T$ are solved. At each iteration, 'areas' of the state space more likely to occur are identified and stored for use during the backward pass calculation when the sub-problem approximation is constructed. By identifying such areas of interest and focusing solution search around areas of the state space more likely to contain the optimal solution, naïve discretization of the state space is avoided and significant computational savings are achieved. Each run of the forward pass involves the sampling of different values of the stochastic variables. Since the main purpose of the forward pass is to drive the solution process towards 'interesting' areas of the state space, a multitude of samples $k=\{1, \ldots, N K\}$ can be obtained and solved in parallel at each iteration, leading to further computational benefits. Constraints (2) - (4), (6) - (7), (9) - (12) and (15) constitute the master problems solved for each period, while (5) and (8) are added at period $T$.

\section{Backward Pass}

The next step of the simulation process is the backward pass, where the sub-problems for periods $T$ to 2 are successively solved (period 1 is associated solely to a master problem). The backward pass aims to gradually improve the approximation of the future cost functions. Bender's cuts approximating each sub-problem's objective function are constructed for the areas of the state space identified during the forward pass. Consequently, the master problem's optimal decisions, which are the values of the state variables corresponding to period $t-1$, are applied and their impact on the optimal sub-problem objective function value is captured through the corresponding dual variables. For each point of interest $k$, the backward pass is solved for all the $s=$ $\{1, \ldots, N S\}$ different samples of the stochastic variables, leading to $k \cdot s$ problems for each period. In order to alleviate the computational burden that arises due to the large number of problems, the sub-problems referring to the same timeperiod can be solved in parallel. The sub-problems consist of all the constraints introduced for the master problems, augmented by constraints (16) - (17), where the auxiliary variables $\tilde{e}_{t, b, s}$ and $\tilde{e}_{t, f, s}^{a g g}$ are introduced and used for the calculation of the dual variables associated with the subproblem's state variables. After solving all the sub-problems referring to period $t$, the respective cuts for each point of interest $k$ are built as per (15), where $\alpha_{t, k}^{i-1}, \lambda_{t, b, i-1, k}^{E}$ and $\lambda_{t, f, i-1, k}^{D}$ are calculated from (18) - (20). The derived cuts are appended to the master problem pertaining to period $t-1$.

$$
\begin{array}{rlr}
\tilde{e}_{t, b, s}=e_{t, b, i-1, k} & : \lambda_{t, b, s}^{E} & \forall t, b, s \\
\tilde{d}_{t, f, s}^{a g g}=d_{t, f, i-1, k}^{a g g} & : \lambda_{t, f, s}^{D} & \forall t, f, s \\
\lambda_{t, b, k}^{E}=\sum_{s} \lambda_{t, b, s}^{E} / N S & & \forall t, b, k \\
\lambda_{t, f, k}^{D}=\sum_{s} \lambda_{t, f, s}^{D} / N S & \forall t, f, k \\
\alpha_{t, k}=\sum_{s} \alpha_{t, s} / N S & \forall t, k
\end{array}
$$

\section{Convergence Criteria}

Iterations between the forward and backward passes continue until an accurate approximation of the future cost functions has been built and an optimal solution of a target quality has been reached. More specifically, upper and lower bounds of the optimal solution are defined and calculated at each iteration, and the algorithm terminates when the distance between these bounds is within a tolerance value. The upper and lower are bounds are given by (21) - (22), and convergence of the two bounds is checked at the end of each forward pass run, according to (23), as in [5].

$$
\begin{gathered}
\underline{z}_{i}=C_{1, i}+\alpha_{1, i} \\
\bar{z}_{i}=\sum_{t, k} C_{t, k} / N K \\
\bar{z}_{i}-(1.96 \cdot \sigma / \sqrt{ } N S) \leq \underline{z}_{i} \leq \bar{z}_{i}+(1.96 \cdot \sigma / \sqrt{ } N S)
\end{gathered}
$$

\section{E. Extension to Time-Dependent Uncertainty}

SDDP has been mainly used for addressing large stochastic problems, under the assumption that the temporal evolution of the uncertain variable is time-independent. In this section we incorporate more information regarding the uncertain variable at hand (in this case the time dependency of wind power outputs) instead of solely relying on a limited set of realizations (whose probability is independent to the stochastic process's history), can provide a more optimal result and contribute towards well-informed decision making.

The proposed algorithm can accommodate the introduction of uncertain variables with temporal dependency, as long as the linearity of the stochastic problem is preserved, so that the computation of the required dual variables is feasible. Consequently, an $n^{\text {th }}$ order autoregressive model, as expressed in (24), has been selected for capturing the stochasticity related to uncertain wind outputs, since it can provide a linear representation of the uncertain variable, while being capable of accurately capturing temporal correlation.

$$
P_{t, w}^{\text {wind }}=\sum_{n} \varphi_{n, w} \cdot P_{t-n, w}^{\text {wind }}+\varepsilon_{t, w} \quad \forall w
$$

In order to accommodate the introduction of the AR model, the state space of the problem needs to be expanded and previous realizations of the stochastic variable, which explicitly affect the current one, become state variables, while future cost functions are approximated taking into account the stochastic process history. Consequently, (24) is added to both the master and sub-problems and sampling of the error term 
$\varepsilon_{t, w}$ provides the different wind output realizations. The expansion of the state space with variables $P_{t-1, w}^{\text {wind }}$ to $P_{t-n, w}^{\text {wind }}$ (where $n$ is order of the AR model) necessitates the introduction of additional dual variables related to the previous wind realizations. In addition, the cuts approximating the future cost function are expanded to capture the impact of the new state variables on the future cost, as shown in (25) - (27).

$$
\begin{aligned}
& a_{t}^{i}\left(e_{t, b}, d_{t, f}^{a g g}, P_{w, t-1}^{\text {wind }}, \ldots, P_{w, t-n}^{\text {wind }}\right) \\
& \geq a_{t, k}^{i-1}\left(e_{t, b, i, k}, d_{t, f, i, k}^{a g g}, P_{w, t-1, i, k}^{\text {wind }}, P_{w, t-n, i, k}^{\text {wind }}\right) \\
& +\sum_{t, \lambda_{t, b, i, k}^{E} \cdot\left(e_{t, b}-e_{t, b, i, k}\right)} \lambda^{b} \sum_{f} \lambda_{t, f, i, k}^{D} \cdot\left(d_{t, f}^{a g g}-d_{t, f, i, k}^{a g g}\right) \\
& +\sum_{w, n} \lambda_{t, w, i, k}^{w n} \cdot\left(P_{t-n, w}^{\text {wind }}-P_{t-n, w, i, k}^{\text {wind }}\right) \\
& \tilde{P}_{t-1, w, s}^{\text {wind }}=P_{t-1, w, i, k}^{\text {wind }} \quad: \lambda_{t, w, i, s}^{w 1} \quad \forall t, w, k \\
& \tilde{P}_{t-n, w, s}^{\text {wind }}=P_{t-n, w, i, k}^{\text {wind }} \quad: \lambda_{t, w, i, s}^{w n} \quad \forall t, w, k
\end{aligned}
$$

We should emphasize that $P_{t-1, w}^{\text {wind }}$ to $P_{t-n, w}^{\text {wind }}$ are known quantities, where the sub-problem for period $t$ is solved, since the uncertainty for this stage has been resolved, so the complexity of the respective cut is not increased. It should also be noted that, even though an AR model has been selected in our case, the proposed modification can be applied for any linear uncertainty model in a straightforward way.

\section{CASE Study}

\section{A. Case study description}

In this section a case study, where the proposed algorithm is applied to the problem of a DER aggregator, which coordinates a flexible load, a battery, a wind turbine and an inflexible load, is presented. The aggregator must come up with the optimal decision set for dispatching its available flexible resources, while facing uncertainty regarding the available wind power output. The study parameters are shown in Table I. The peak demand of the aggregator's portfolio is $400 \mathrm{~kW}$ when the flexibility option is not exercised, while the maximum power that can be imported from the grid is set to $400 \mathrm{~kW}$ as well, so that there is always sufficient demand supply, even in cases of wind scarcity. The battery size, charging and discharging power ratings have been selected such that the battery can cover the biggest part of the total demand for a period and even accommodate available wind power outputs at periods of wind power abundance. We should note that the option of exporting power to the grid is not available, so that the battery is not used for energy arbitrage with the upstream system. This is done for simplicity, in order to focus solely on the battery's role in wind management. A planning horizon of 6 hours is studied, since wind power output forecasting beyond that horizon is not considered sufficiently accurate and the battery's energy capacity (and round-trip efficiency) is not large enough to warrant consideration of strategic charging/discharging beyond a few hours ahead of need.

In order to build a realistic wind output model, historical values of wind power output in the Northern UK area for a representative month have been obtained from [12]. The time dependency of the uncertain wind output is captured through a lag-1 autoregressive model as shown in (24). The performance of the proposed SDDP model is evaluated against a traditional scenario tree approach for modelling uncertain variables. An $\operatorname{ARIMA}(5,1,2)$ model has been fit to the historical wind power

\begin{tabular}{|c|c|c|c|}
\hline \multirow{2}{*}{\multicolumn{4}{|c|}{$\begin{array}{l}\text { E I. CASE STUDY PARAMETERS } \\
\text { Energy Storage Parameters }\end{array}$}} \\
\hline & & & \\
\hline & $E_{b}^{\max } / E_{b}^{\min }$ & \multirow{2}{*}{\multicolumn{2}{|c|}{$\begin{array}{c}700 \mathrm{kWh} / 140 \mathrm{kWh} \\
280 \mathrm{~kW}\end{array}$}} \\
\hline & $P_{b}^{S}$ & & \\
\hline & $E_{0, b}$ & \multicolumn{2}{|c|}{$\begin{array}{c}280 \mathrm{~kW} \\
350 \mathrm{kWh}\end{array}$} \\
\hline & $\eta_{b}$ & \multicolumn{2}{|c|}{$\begin{array}{l}350 \mathrm{kWh} \\
09\end{array}$} \\
\hline \multicolumn{4}{|c|}{ Flexible Load Parameters } \\
\hline & $\overline{D_{t, f}^{\text {base }}}$ & \multicolumn{2}{|c|}{$200 \mathrm{~kW}$} \\
\hline & $S_{f}$ & \multicolumn{2}{|c|}{$10 \%$} \\
\hline \multicolumn{4}{|c|}{ System Parameters } \\
\hline & $P_{t, w}^{\text {wind }} / P_{t}^{g, \text { sell }}$ & \multicolumn{2}{|c|}{$1000 \mathrm{~kW} / 0 \mathrm{~kW}$} \\
\hline & $P_{t}^{t, w}$,buy $t P^{\text {gen }}$ & \multicolumn{2}{|c|}{$400 \mathrm{~kW} / 100 \mathrm{~kW}$} \\
\hline & ${ }^{t} D_{t}^{\text {inf }}$ & \multicolumn{2}{|c|}{$200 \mathrm{~kW}$} \\
\hline & $C_{t}^{\text {grid }}, C_{t}^{\text {gen }}$ & \multicolumn{2}{|c|}{$0.15 £ / \mathrm{kWh} / 1 £ / \mathrm{kWh}$} \\
\hline \multicolumn{4}{|c|}{ SCENARIO TREES STRUCTURE } \\
\hline $\begin{array}{l}\text { Complexity } \\
\text { Level }\end{array}$ & $\begin{array}{c}\text { Structure } \\
\text { (nodes per stage) }\end{array}$ & $\begin{array}{l}\text { Number of } \\
\text { nodes }\end{array}$ & $\begin{array}{l}\text { Number of } \\
\text { scenarios }\end{array}$ \\
\hline $\mathrm{C} 1$ & $1-1-1-1-1-1$ & 6 & 1 \\
\hline $\mathrm{C} 2$ & $1-2-2-2-2-2$ & 11 & 2 \\
\hline $\mathrm{C} 3$ & $1-2-4-6-8-10$ & 31 & 10 \\
\hline $\mathrm{C} 4$ & $1-2-4-8-12-16$ & 43 & 16 \\
\hline $\mathrm{C} 5$ & $1-2-4-8-16-32$ & 63 & 32 \\
\hline C6 & $1-3-9-27-81-243$ & 364 & 243 \\
\hline
\end{tabular}
output data and then a sufficient number of scenarios have been sampled and used both for fitting the AR(1) model and constructing scenario tree representations of varying degrees of complexity. As presented at Table II, six scenario trees were constructed according to a scenario reduction process based on Kantorovich distance [13].

In order to compare the solution efficiency and quantify the expected benefit of the seven models, out-of-sample Monte Carlo validation is used. In this framework, 10 wind power starting points are considered $(5 \%, 15 \%$, up to $95 \%$ of $P_{1}^{\text {wind }}$ ) to (i) construct scenario of different complexity as shown below (ii) identify the optimal first-stage decisions for all six scenario tree models and the SDDP model. Subsequently, for each starting point, 1000 6-hour scenario paths are sampled from the original ARIMA model and each scenario is solved to optimality. In this way we can compute the impact that an under-informed first-stage decision can have on subsequent operation; note that this provides a lower bound to the potential benefit of using a more advanced method since perfect information is assumed from $t=2$ onwards. The modelling was carried out in MATLAB R2015a and FICO Xpress [14], on a $3.33 \mathrm{GHz}$ Intel Xeon computer.

\section{B. Results}

Figure 1. illustrates the expected monthly aggregator cost for the different scenario tree complexities and the SDDP model. It can be observed that the solutions obtained by the SDDP model outperform the first 2 scenario tree types by $8.5 \%$. The third tree type exhibits a significant decrease in the expected cost, implying that 10 scenarios capture more precisely the problem uncertainty, but still SDDP reduces cost 
by $1.5 \%$. It is only after $\mathrm{C} 5$, that the solutions obtained from the trees outperform the ones obtained from SDDP, but still the difference not significant. It should be mentioned that the simulation times for the SDDP model are equivalent to the simpler scenario tree types, while it is almost half of the most complex one. The computational benefit is even more pronounced if the planning horizon is increased.

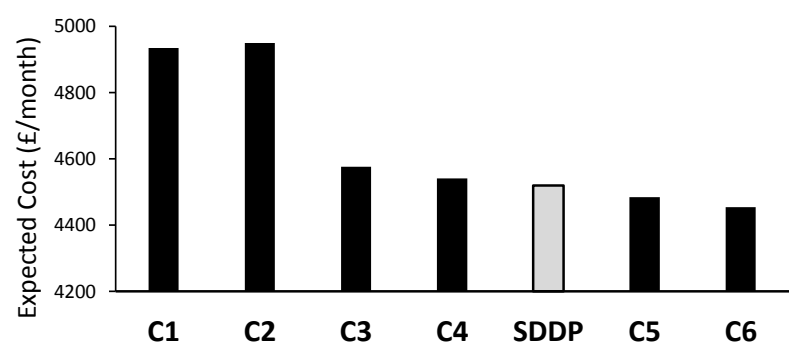

Figure 1. Expected cost for SDDP and different scenario tree types.

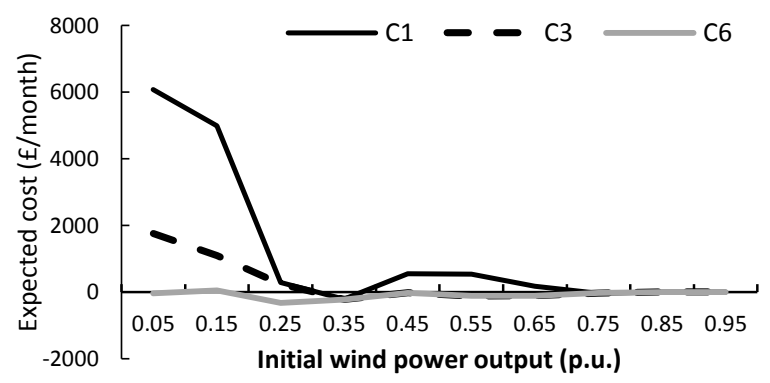

Figure 2. Difference in expected cost between SDDP and different scenario trees for different initial wind power output.

Figure 2. depicts the comparison of the expected cost between the SDDP model and the $1^{\text {st }}, 3^{\text {rd }}$ and $6^{\text {th }}$ scenario tree structures for each wind output starting point, where positive (negative) values signify that SDDP outperforms (underperforms) the scenario tree solution for the specific wind output. We should notice that SDDP performs significantly better than trees for almost all starting points. In general, benefits are augmented for low wind starting points, while they diminish when we proceed towards the last ones. This is an expected outcome, as low wind starting points means that most probably less wind will be available in the operating window, rendering the quality of the forecast important and increasing the impact of strategic decisions regarding initial charging/discharging. On the other hand, for the high-wind starting points, there is abundance of wind and whichever decision is made at the beginning of the period has minor impact on the aggregator's expected cost.

\section{CONCLUSION}

This paper presents an algorithm for the optimal operation of a DER aggregator under uncertain wind power output, while taking into account the temporal dependency of the uncertain variable. An extension of the SDDP algorithm, where an AR model is incorporated has been presented and analyzed. This approach does not require discretization of neither the state space nor the uncertain variables, thus eliminating problems that would emanate due to the "curse of dimensionality'. The proposed approach has been showcased for a representative DER aggregator portfolio, which consists of an ES unit, a flexible load, a wind turbine and an inflexible load and has been compared to scenario trees with different complexity levels. The proposed approach exhibits significant benefits compared to scenario trees of low and medium complexity, which are significantly enhanced for low wind outputs. The benefits seem to diminish at cases of wind abundance, when available wind can satisfy the demand needs independently of the aggregator's decision making process.

The future goals of this work evolve in two axes. The first one constitutes the further enhancement of the algorithm with more complex uncertainty models, which will be able to capture the temporal dependencies of more than one uncertain variables and the inter-dependencies among them. The second goal comprises the expansion of the aggregator portfolio, so that it will include more assets, so that we could study the impact of different portfolio compositions and flexibility levels on the optimal aggregator strategy.

\section{REFERENCES}

[1] Smart Grid Task Force, "Regulatory Recommendations for the Deployment of Flexibility: EU SGTF-EG3 Report," 2015.

[2] P. Balram, A. T. Le and L. Bertling Tjernberg, "Stochastic programming based model of an electricity retailer considering uncertainty associated with electric vehicle charging," 2013 10th International Conference on the European Energy Market (EEM), Stockholm, pp. 1-8, 2013.

[3] D. T. Nguyen and L. B. Le, "Risk-Constrained Profit Maximization for Microgrid Aggregators With Demand Response," IEEE Transactions on Smart Grid, vol. 6, no. 1, pp. 135-146, Jan. 2015.

[4] H. A. Aalami and S. Nojavan, "Energy storage system and demand response program effects on stochastic energy procurement of large consumers considering renewable generation," IET Generation, Transmission \& Distribution, vol. 10, no. 1, pp. 107-114, July 2016.

[5] A. Bhattacharya, J. Kharoufeh and B. Zeng, "Managing Energy Storage in Microgrids: A Multistage Stochastic Programming Approach," IEEE Transactions on Smart Grid, accepted for publication.

[6] H. Chen, H. Li, R. Ye and B. Luo, "Robust scheduling of power system with significant wind power penetration," 2012 IEEE Power and Energy Society General Meeting, San Diego, CA, pp. 1-5, 2012.

[7] M. Rahimiyan, L. Baringo and A. J. Conejo, "Energy Management of a Cluster of Interconnected Price-Responsive Demands," IEEE Transactions on Power Systems, vol. 29, no. 2, pp. 645-655, March 2014.

[8] Z. Chen, L. Wu and Y. Fu, "Real-Time Price-Based Demand Response Management for Residential Appliances via Stochastic Optimization and Robust Optimization," IEEE Transactions on Smart Grid, vol. 3, no. 4, pp. 1822-1831, Dec. 2012.

[9] T. Summers, J. Warrington, M. Morari and J. Lygeros, "Stochastic optimal power flow based on convex approximations of chance constraints," Power Systems Computation Conference (PSCC), Wroclaw, pp. 1-7, 2014.

[10] M. V. F. Pereira and L. M. V. G. Pinto, "Multi-stage stochastic optimization applied to energy planning," Mathematical Programming, vol. 52, pp. 359-375, 1991

[11] M. V. F. Pereira, "Optimal stochastic operations scheduling of large hydroelectric systems," International Journal of Electrical Power \& Energy Systems, vol. 11, pp. 161-169, 1989.

[12] I. Staffell, S. Pfenninger, "Using bias-corrected reanalysis to simulate current and future wind power output", Energy, vol. 114, pp 12241239, Nov 2016.

[13] N. Growe-Kuska, H. Heitsch and W. Romisch, "Scenario reduction and scenario tree construction for power management problems," Power Tech Conference Proceedings, 2003 IEEE Bologna, vol.3, 2003.

$\begin{array}{lll}\text { [14] FICO Xpress } & \text { Optimization } \\ \text { http://www.fico.com/en/products/fico-xpress-optimization-suite. }\end{array}$
Suite, 